\title{
Actualización de la distribución y redescripción de Entomochilus nitens Kulzer, 1956 (Coleoptera: Tenebrionidae: Physogasterini)
}

\section{Distribution update and redescription of Entomochilus nitens Kulzer, 1956 (Coleoptera: Tenebrionidae: Physogasterini)}

\section{Marcos Ferrú ${ }^{1,2 *}$ \& Margarita Ruiz de Gamboa ${ }^{3}$}

\author{
'Universidad Católica de la Santísima Concepción, Alonso de Ribera 2850, Concepción, Chile. \\ ${ }^{2}$ Centro de Muestreo y Análisis Biológico (CEMABIO), Valle Central \# 3240 Iquique, Chile. \\ ${ }^{3}$ Centro de Investigación en Medio Ambiente (CENIMA), Universidad Arturo Prat, Casilla 121, Iquique, Chile. \\ *E-mail: marcos.ferru@gmail.com
}

\begin{abstract}
RESUMEN
Entomochilus nitens Kulzer, 1956 (Tenebrionidae: Physogasterini) es una especie de escarabajo cuya descripción morfológica original es poco detallada y existen escasos registros geográficos. Aquí se redescribe a esta especie, detallando la genitalia del macho, carácter hasta ahora desconocido. Se entrega una nueva localidad de su distribución en el norte de Chile y se reconoce por primera vez su presencia para Perú.

Palabras clave: Chile, Entomochilus, Perú, redescripción.
\end{abstract}

\section{ABSTRACT}

Entomochilus nitens Kulzer, 1956 (Tenebrionidae: Physogasterini) is a beetle species that has a vague morphological description and few geographical records. We redescribe this species, detailing the male genitalia, character unknown until now. We give new locality for Northern Chile, and here we indicate this species for Peru for the first time.

Keywords: Chile, Entomochilus, Peru, redescription.

La tribu Physogasterini pertenece a la familia Tenebrionidae y está actualmente compuesta por los géneros Entomochilus Solier, 1844, Phylorea Erichson, 1834, Physogaster Guérin-Méneville, 1834, Physogasterinus Kaszab, 1981 y Pimelosomus Burmeister, 1875. Esta tribu es endémica de Sudamérica y cuenta actualmente con 64 especies (Ferrú 2016).

El género Entomochilus con 20 especies (Vidal \& Guerrero 2007), es el más numeroso de la tribu Physogasterini y según la literatura, es endémico de Chile (Peña 1980). Este género presenta un reducido rango geográfico de distribución, entre los $18^{\circ}$ y $34^{\circ}$ de latitud sur (Vidal \& Guerrero 2007), desde el nivel del mar hasta los 3.500 m de altitud (Peña 1980; Cepeda 1997), en las provincias biogeográficas de la Puna, Atacama, Coquimbo y Santiago (Morrone 2014).
Entomochilus nitens Kulzer, 1956 es una especie glabra señalada sólo para Chile, con escasos registros de localidades conocidas. Kulzer (1956) realiza una breve descripción morfológica de E. nitens, pero sin imagen, dibujo, ni mapa de distribución de la especie; señala varias localidades, donde sólo la primera es asociada a un país (Arica, Alcérreca, Chile), para el resto sólo hace mención del nombre de la localidad y cantidad de ejemplares de cada una. Posteriormente, Peña (1980) incluye a esta especie en su clave taxonómica del género Entomochilus y señala que se distribuye en la cordillera de Tarapacá, que posteriormente fue dividida en las actuales Región de Arica y Parinacota, y Región de Tarapacá. Vidal \& Guerrero (2007) la nombran como parte de un listado sin aportar nuevos antecedentes. Finalmente, Ferrú \& Elgueta (2011) realizan nuevos aportes de distribución para el género 
Entomochilus para las regiones Arica y Parinacota y Tarapacá, donde se incluye a esta especie.

Recientes registros de campo permiten entregar nuevas localidades de Entomochilus nitens en Chile y la revisión de material tipo depositado en Naturhistorisches Museum Basel (Suiza) permite reconocer la distribución de esta especie y del género Entomochilus al Perú. Por lo anterior, el objetivo de este trabajo es actualizar la distribución geográfica y entregar una descripción más detallada de E. nitens.

Para realizar la redescripción de Entomochilus nitens y definir su distribución geográfica, se realizó una búsqueda bibliográfica para obtener la descripción original (Kulzer 1956), además de todos los trabajos que hacen referencia a esta especie (Peña 1980; Vidal \& Guerrero 2007). Por otra parte, se revisó material de diversas colecciones: Museo Nacional de Historia Natural de Santiago de Chile (MNNC), Natural History Museum Entomology Basel (NMB) y material propio (Centro de Muestreo y Análisis Biológico (CEMABIO)). El material fue examinado bajo lupa estereoscópica, excepto el material tipo depositado en NMB, el cual fue estudiado mediante fotografías de alta resolución (Fig.1).

\section{REDESCRIPCIÓN}

Entomochilus nitens Kulzer, 1956 (Fig. 1 - 3)

Entomochilus nitens Kulzer, 1956: 496, 497; Peña, 1980: 51

Diagnosis: Cuerpo convexo, de color negro brillante, tarsos y antenas también negras brillantes. Superficie glabra, frente con gránulos reniformes y con cerdas en su fracción inferior, protórax con puntuación separada en el centro, la que tiende a fusionarse hacia los costados, élitros levemente rugosos, con puntuación separada y claramente notoria.

Medidas: Longitud: 9,9 a 10,4 mm; Ancho: 5,8 a 7,2 mm; $(n=10)$

Cabeza (Fig. 1c): ligeramente redonda, ojos reniformes. Mejillas fuertemente redondeadas en los lados, ligeramente levantadas. Clípeo corto, el borde anterior es arqueado, con puntuación escasa que está asociada a la sutura clipeal, la que es transversal, ligeramente curvada, muy profunda y estrecha. Vertex con gránulos más pequeños que la frente, el tamaño de esta granulosidad tiende a aumentar hacia el centro de la frente, acompañada de pilosidad corta (Fig. 1c), las antenas dirigidas hacia atrás sobrepasan el pronoto desde el octavo terjito.

Tórax: Disco pronotal más ancho en su parte media, ángulos anteriores agudos, ángulos posteriores completamente redondeados. Con presencia de margen lateral (Flores 2000; Vidal \& Guerrero 2007; Ferrú 2016). Puntuación ovalada separada por un espacio del diámetro de un punto en su mayor parte, viéndose muchos puntos unidos longitudinalmente pero distinguibles, la puntuación tiende a aumentar de tamaño hacia los costados (Fig. 1a, 1b, 2a, 2b). Prosterno conproceso prosternal pequeño, presente anteriormente, más amplio en el margen anterior. Metaesterno granulado en los lados.

Élitros: ovalados Superficie finamente rugosa, con puntuación clara y definida, principalmente en la mitad anterior (Fig. 1d, 2c), con gránulos claros en el margen lateral, sin pilosidad. Epipleura presente, lisa, sin pilosidad, con gránulos separados.

Abdomen: Finamente punteado.

Patas: Brillantes, fémures con puntuación marcada, acompañada de cerdas espiniformes de pequeño tamaño, tibias con gránulos que presentan pequeñas cerdas espiniformes, rojas y cortas.

Genitalia del macho: Edeago (Fig. 2d) con parámeros ovalados, de ápices redondeados, la escotadura media ocupa la quinta parte de los parámeros. En la vista lateral se observa levemente curvo, la base es cóncava. Lóbulo medio transparente y más ancho en su fracción media (Fig. 2d), la fracción anterior es convexa y la fracción posterior presenta una concavidad.

Material tipo: Holotipo [PERÚ, (Tacna: Pachia), Apachetas de Huailillas, 4.200 m, 2102-1948, Leg. Kuschel], (NMB). Alotipo [CHILE (Parinacota, General Lagos), Alcérreca, 2202-1948, 3.938 m. Leg: Kuschel] (NMB). 7 Paratipos [CHILE (Parinacota, Parinacota), General Lagos, Alcérreca: 22-021948, Leg: Kuschel] (NMB). 7 Paratipos (MNNC). 1 Paratipo [CHILE (Antofagasta, El Loa), San Pedro de Atacama, Queipiaco, 03-06-1952, Leg: L. Peña] (CLP).

Material estudiado: 1 Paratipo [CHILE Arica y Parinacota, Alcérreca, 22-02-1948, 4.000 m. Leg. Kuschel] (MNNC); 1 Paratipo [CHILE, Arica y Parinacota, Alcérreca, Tacora, 1007-1952, 4.085 m, L. Peña] (MNNC); 6 ejemplares [CHILE (Arica y Parinacota, Alcérreca, Visviri) 23/24-11-2007, Leg. M. Ferrú \& J. Guzmán] (CEMABIO); 3 ejemplares [CHILE, Tarapacá, Tamarugal, Pica, Cariquima, 20-11-2007, Leg. M. Ferrú] (CEMABIO); 4 ejemplares [CHILE, Tarapacá, Tamarugal, Pica, Collahuasi 28/29-9-2002, Leg. C. Rojas] (CEMABIO); 3 ejemplares [CHILE, Tarapacá, Tamarugal, Pica, Collahuasi, 24-01-2003, Leg. M. Ferrú] (CEMABIO). 3 ejemplares [CHILE, Tarapacá, Tamarugal, Colchane, Quebrada Tacore, 02-12-2007, Leg. M. Ferrú] (CEMABIO). 

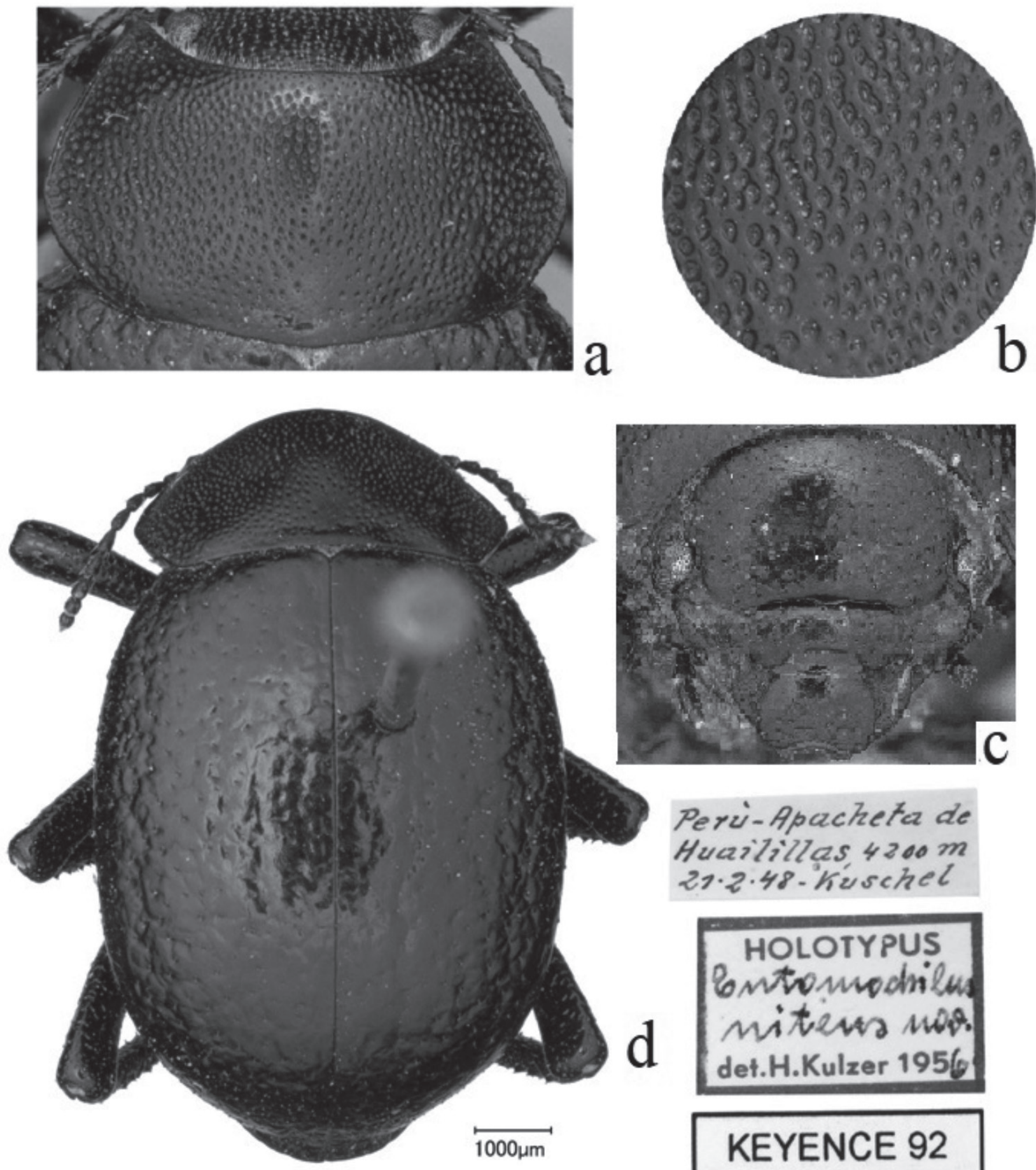

\section{KEYENCE 92}

Figura 1. Holotipo de Entomochilus nitens y sus etiquetas. (a) Vista dorsal del Tórax, (b) de Puntuación lateral del pronoto, (c) habitus, (d) Puntuación en la cabeza. / Holotype of E. nitens and its vouchers. (a) Thorax dorsal view, (b) Pronotum punctuation over lateral area, (c) habitus, (d) Head punctuation. 

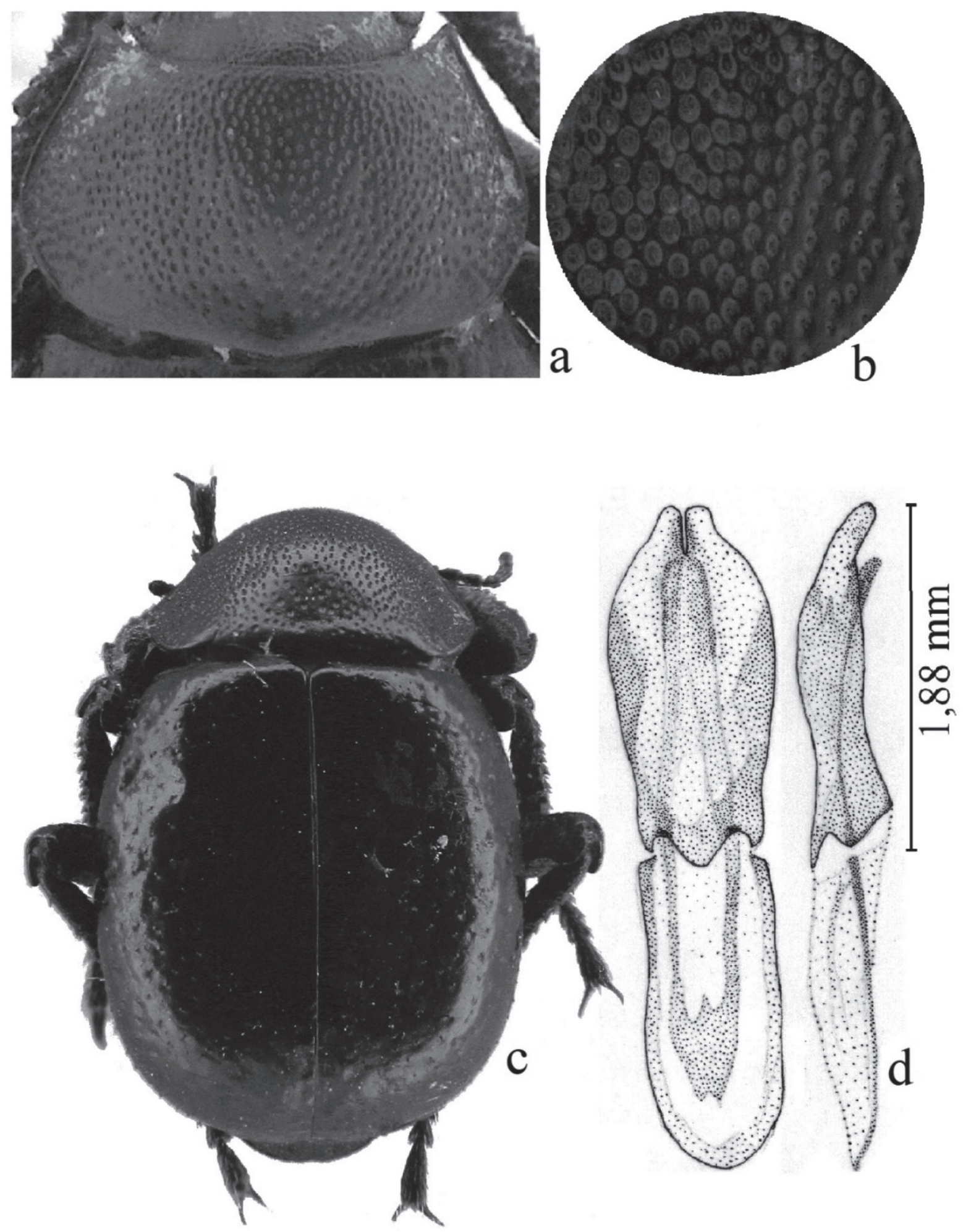

Figura 2. Entomochilus nitens de Visviri (Chile). (a) Vista dorsal del tórax, (b) Puntuación lateral del pronoto, (c) Habitus, (d) Vista lateral y ventral del edeago. / E. nitens from Visviri (Chile). (a) Thorax dorsal view, (b) Pronotum over lateral area, (c) Habitus, (d) Edeagus lateral and ventral view. 
Distribución geográfica (Fig. 3): PERÚ: Región de Tacna: Departamento de Tacna, Municipalidad de Pachia: localidad de Apachetas de Huailillas $\left(-17,7689^{\circ},-69,8389^{\circ}, 4.457 \mathrm{~m}\right.$. CHILE: Región de Arica y Parinacota: Provincia de Parinacota: Comuna de General Lagos: localidad de Alcérreca $\left(-17,9835^{\circ}\right.$, $\left.-69,6501^{\circ}, 3.924 \mathrm{~m}\right)$, Ancolacani $-17,8502^{\circ},-69,6001^{\circ}$, $4.032 \mathrm{~m})$, Tacora $\left(-17,7668^{\circ},-69,7168^{\circ}, 4.000 \mathrm{~m}\right)$ y Visviri $\left(-17,58345^{\circ},-69,46676^{\circ}, 4.083\right.$ m); Región de Tarapacá: Provincia del Tamarugal: Comuna de Colchane, Localidad de Cariquima $\left(-19,4668^{\circ},-68,6335^{\circ}, 3.765 \mathrm{~m}\right)$ y Quebrada Tacore $\left(-18,5001^{\circ},-69,6668^{\circ}, 3.980 \mathrm{~m}\right)$; Región de Tarapacá: Provincia del Tamarugal: Comuna de Pica, Collahuasi $\left(-20,9669^{\circ},-68,6835^{\circ}, 3.911 \mathrm{~m}\right)$.

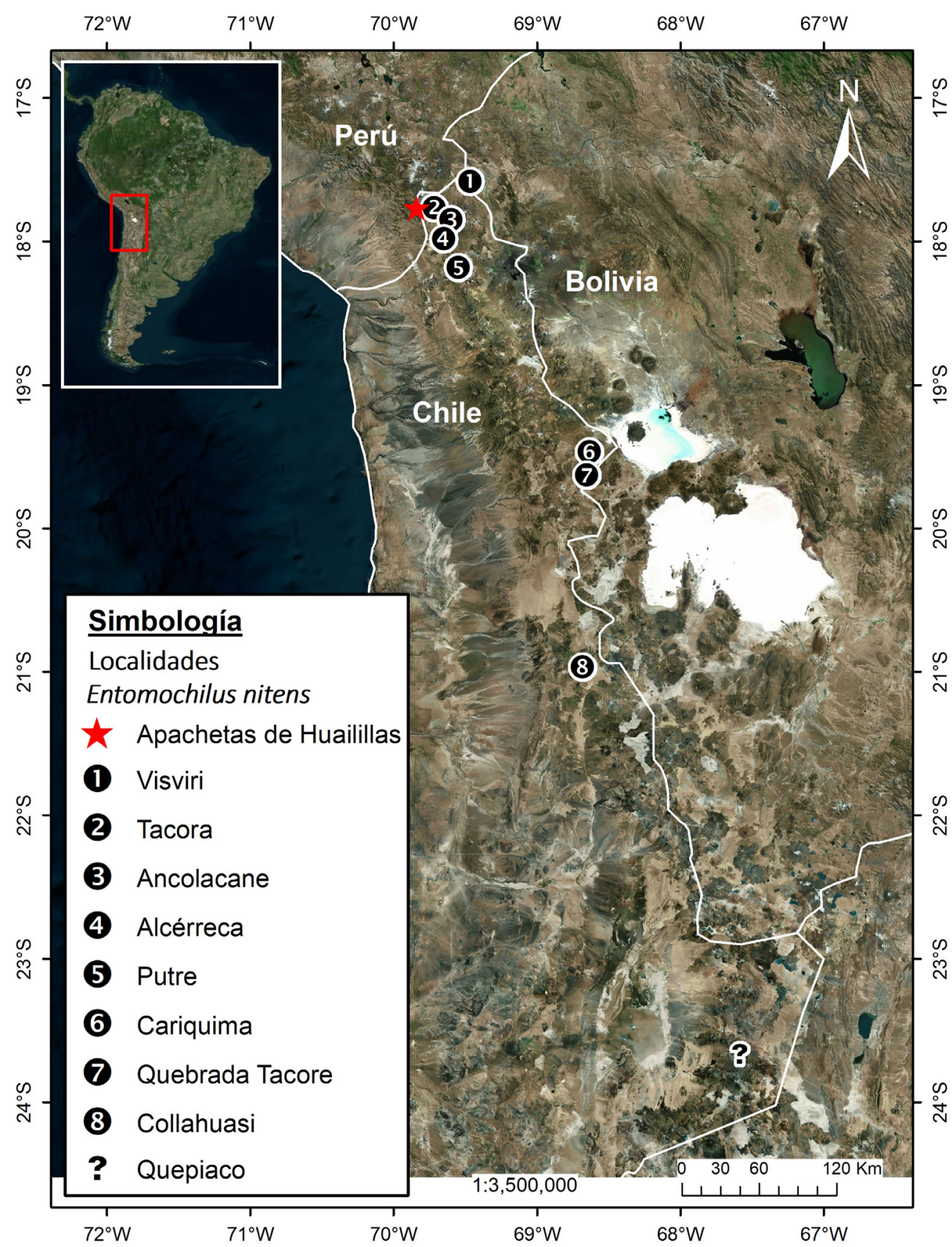

Fıgura 3. Mapa de distribución de localidades conocidas para Entomochilus nitens. La estrella roja es la localidad tipo y Quebrada Tacore es un nuevo registro de distribución. / Distribution map of known locations to E. nitens. Red star is type locality and Quebrada Tacore is a new record. 
Kulzer (1956) señala a esta especie para la localidad de Quepiaco (Región de Antofagasta), lo que es repetido por Peña (1966) y Ferrú \& Elgueta (2011). Aquí, este registro es considerado dudoso ya que (i) se ubica a $300 \mathrm{~km}$ de distancia (en línea recta) de la localidad más al sur confirmada en este trabajo (Collahuasi, Región de Tarapacá), (ii) en numerosas expediciones, los autores no han registrado a Entomochilus nitens en la Región de Antofagasta, pero (iii) sí se han registrado otras especies del género (e.g. Entomochilus rugosus y E. varius). Por lo anterior, el registro de Quepiaco debe ser confirmado. No obstante, en este trabajo no se logró obtener información sobre ese material.

La distribución geográfica de las especies del género Entomochilus era sólo conocida para Chile, por lo que se consideraba endémico de este país. En este trabajo se constató que el Holotipo de E. nitens pertenece a una localidad del Departamento de Tacna, extremo sur de Perú. Por lo anterior, el género Entomochilus ya no es endémico de Chile y E. nitens debe ser incluida como parte de la fauna del Perú. Lo anterior, sumado a que esta especie habita en Visviri (Fig. 3), poblado más septentrional de Chile, hito tripartito limítrofe con Perú y Bolivia, permite inferir la posibilidad de que esta especie pueda ser encontrada en nuevas localidades de la cordillera del Perú y potencialmente también en Bolivia, ya que presenta una marcada distribución andina (Fig. 3).

\section{AGRADECIMIENTOS}

A Mario Elgueta del Museo Nacional de Historia Natural de Chile, por sus comentarios que permitieron mejorar este trabajo y permitir el acceso del primer autor a la colección entomológica del Museo Nacional de Historia Natural de Chile (MNNC) para la observación del material tipo del género Entomochilus; un agradecimiento especial a Mathias
Borer del Natural History Museum Entomology Basel (NMB), por el acceso y envío de los fototipos de E. nitens, proporcionados con mucha amabilidad y buena disposición.

\section{REFERENCIAS}

Cepeda, J. 1997. Insectos de la alta montaña del valle de Elqui. Ediciones Dirección de Investigación y Desarrollo, Universidad de La Serena, Chile. 215 pp.

Ferrú, M., Elgueta, M. 2011 Lista de Coleópteros (Insecta: Coleoptera). de las regiones de Arica y Parinacota y de Tarapacá, Chile. Boletín del Museo Nacional de Historia Natural, Chile 60: 9-61.

Ferrú, M. 2016. Posición sistemática de Entomochilus parvus (Solier, 1851) (Coleoptera: Tenebrionidae: Physogasterini). IDESIA 34(1): 101-104.

Flores, G.E. 2000. Cladistic Analysis of the Neotropical Tribe Nycteliini (Coleoptera: Tenebrionidae). Journal of the New York Entomological Society 108(1-2): 13-25.

Kulzer, H. 1956. Bemerkenswerte Tenebrioiniden aus der TharWüste. 14. Beitrag zur Kenntnis der Tenebrioniden (Leg.). Entomologische Arbeiten Munich 7: 895-965.

Morrone, J.J. 2014. Biogeographical regionalisation of the Neotropical region. Zootaxa 3782(1): 1-110.

Peña, L.E. 1980. Aporte al conocimiento de los Tenebrionidos de América del Sur. Revista Chilena de Entomología 10: 49-59.

Smith, A., Mendoza, A., Flores, G., Aalbu, R. 2015. Beetles (Coleoptera) of Peru: A Survey of the Families. Tenebrionidae. Journal of the Kansas Entomological Society 88(2): 221-228.

Vidal, P., Guerrero, M. 2007. Los Tenebriónidos de Chile. Ediciones Universidad Católica de Chile, Santiago. 478 pp.

Received: 23.09.2019

Accepted: 26.06.2020 